\title{
QUASI-UNMIXED LOCAL RINGS AND QUASI-SUBSPACES
}

\author{
PETER G. SAWTELLE ${ }^{1}$
}

\begin{abstract}
The concept of a quasi-subspace is defined so that it plays a role relative to quasi-unmixedness analogous to that of subspace to unmixedness. This definition is used to characterize quasi-unmixed local rings.
\end{abstract}

1. Introduction. In this paper, a ring shall be a commutative ring with identity. The terminology is basically that of [3] and [9]. In particular, a semilocal (Noetherian) ring $R$ is called unmixed (resp., quasi-unmixed) in case depth $p=$ altitude $R$, for every prime divisor (resp., minimal prime divisor) $p$ of zero in the completion of $R$.

Proposition 3.3 in [1] gives an example of a local domain $A$ of altitude two whose integral closure is a convergent power series ring in two variables over the complex number field, and whose completion $A^{*}$ contains an imbedded prime divisor of zero. Thus $A$ is not unmixed. However, by [5, Corollary 3.4(i)], $A$ is quasi-unmixed. ${ }^{2}$ (This example answers Problem 1 of [2, p. 62].)

Ratliff $[6, \S 4]$ characterizes an unmixed local ring $R$ in terms of certain local rings that contain $R$ as a subspace. This paper parallels [6, $\S 4]$; in particular, the concept of a quasi-subspace is introduced to play a role relative to quasi-unmixedness analogous to the role played by a subspace to unmixedness. Since the concepts of unmixedness and quasi-unmixedness are distinct, the results and techniques below should be of assistance in investigating quasi-unmixed local rings. The results of this paper and of [5], [6] have been used in [8] to characterize unmixed and quasiunmixed local domains. (Specifically, if $\mathscr{R}$ is a particular Rees ring of a local domain $R$, then the property that a certain transform ring of $\mathscr{R}$ is contained in the integral closure of $\mathscr{R}$ (resp., is Noetherian) is a condition which characterizes (resp., is closely related to) the quasi-unmixedness (resp., unmixedness) of $R$.)

Received by the editors March 21, 1972.

AMS (MOS) subject classifications (1970). Primary 13H10, 13C15; Secondary $13 \mathrm{~J} 10$.

Key words and phrases. Local ring, unmixed, quasi-unmixed, subspace of a semilocal ring, Rees ring.

${ }^{1}$ This paper is part of the author's doctoral dissertation written at the University of California at Riverside under the direction of Professor Louis J. Ratliff, Jr.

${ }^{2}$ This example was mentioned to the author by Professor Louis J. Ratliff, Jr.

(c) American Mathematical Society 1973 


\section{Preliminary definitions and results.}

Definition 1. Let $R$ and $S$ be semilocal rings with completions $R^{*}$ and $S^{*} . R$ is a quasi-subspace of $S$ if there exists an isolated ideal component $I^{*}$ of zero in $R^{*}$ such that $I^{*} \subseteq \operatorname{rad} R^{*}$ and such that $S^{*}$ dominates $R^{*} / I^{*}$ and $S$ dominates $R / I$, where $I=I^{*} \cap R$.

Note that $I^{*} \subseteq \operatorname{rad} R^{*}$ implies that $I \subseteq\left(\operatorname{rad} R^{*}\right) \cap R=\operatorname{rad} R$, and so $\operatorname{rad} I=\operatorname{rad} R$. Also, by letting $I^{*}=(0)$, note that a semilocal ring is a quasi-subspace of itself and that a semilocal ring that is a subspace of a semilocal ring $S$ is also a quasi-subspace of $S$.

Lemma 2 below gives a characterization of quasi-subspace that is easier to use for the rings considered in $\S 3$. Lemma 3 then shows how the concept of quasi-subspace is related to the minimal prime divisors of zero of these rings. In particular, Lemma 3 and Corollary 8 give a relation between quasi-unmixed local rings and the minimal prime divisors of zero in certain Rees rings of their completions (Corollary 9).

For ease of notation, let $R_{k}$ denote a polynomial ring in $k$ indeterminants over a ring $R$. For the completion $R^{*}$ of $R, R_{k}^{*}=\left(R^{*}\right)_{k}$.

Lemma 2. Let $(R, M)$ be a local ring with completion $\left(R^{*}, M^{*}\right)$. Let $k \geqq 0$, and let $y_{1}, \cdots, y_{d}(d \geqq 0)$ be elements of the total quotient ring of $R_{k}$. Let $A=R_{k}\left[y_{1}, \cdots, y_{d}\right]$ and $A^{*}=R_{k}^{*}\left[y_{1}, \cdots, y_{d}\right]$. Let $P^{*}$ be a prime ideal in $A^{*}$ such that $P^{*} \cap R^{*}=M^{*}$, and let $P=P^{*} \cap A$. Then, $R$ is a quasi-subspace of $A_{P}$ if and only if $A_{P^{*}}^{*}$ dominates $R^{*} / I^{*}$ for some isolated ideal component of zero in $R^{*}$ such that $I^{*} \subseteq \operatorname{rad} R^{*}$.

Proof. Let $A_{P *}^{*}$ dominate $R^{*} / I^{*}$ and $I=I^{*} \cap R$, where $I^{*}$ is given above. Let $K$ (resp., $K^{*}$ ) be the kernel of the natural homomorphism of $A$ into $A_{P^{\prime}}$ (resp., $A^{*}$ into $A_{P *}^{*}$ ). Since $A_{P}=(A / K)_{P / K}$ is a dense subspace of $A_{P *}^{*}=\left(A^{*} / K^{*}\right)_{P^{*} / K^{*}}\left[6\right.$, Lemma 3.2], then $K=K^{*} \cap A$. Also, $I^{*}=$ $K^{*} \cap R^{*}$. Therefore, $I=K \cap R$, and so $R / I$ is a subring of $A_{P}$. Since $P \cap R=M$ [6, Lemma 3.2], $A_{P}$ dominates $R / I$. Since $\left(A_{P}\right)^{*}=\left(A_{P^{*}}^{*}\right)^{*}$ [6, Lemma 3.2], and $\left(A_{P^{*}}^{*}\right)^{*}$ dominates $A_{P^{*}}^{*}$, then $\left(A_{P}\right)^{*}$ must dominate $R^{*} / I^{*}$.

Conversely, let $R$ be a quasi-subspace of $A_{P}$. Let $I^{*}$ be as in Definition 1 , and let $K^{*}$ be as above. Then $R^{*} / I^{*}$ is a subring of $\left(A_{P}\right)^{*}=\left(A_{P^{*}}^{*}\right)^{*}$, and is therefore a subring of $A_{P *}^{*}$. Hence $A_{P *}^{*}$ dominates $R^{*} / I^{*}$, since $P^{*} \cap$ $R^{*}=M^{*}$. Q.E.D.

Lemma 3 (CF. [6, Lemma 4.5(1)]). Let $R, R^{*}, A, A^{*}, P$ and $P^{*}$ be as in Lemma 2. Then $R$ is a quasi-subspace of $A_{P}$ if and only if $P^{*}$ contains all minimal prime divisors of zero in $A^{*}$.

Proof. Let $R$ be a quasi-subspace of $A_{P}$. By Lemma $2, R^{*} / I^{*}$ is a subring of $A_{P^{*}}^{*}$, where $I^{*}$ is given in Definition 1. Thus $I^{*}=K^{*} \cap R^{*}$, 
where $K^{*}$ is given in Lemma 2 . Therefore, since $K^{*}$ is an isolated ideal component of zero in $A^{*}$, and since $A^{*}$ and $R_{k}^{*}$ have the same total quotient ring, it follows that $K^{*} \cap R_{k}^{*}=I^{*} R_{k}^{*} \subseteq\left(\operatorname{rad} R^{*}\right) R_{k}^{*}=\operatorname{rad} R_{k}^{*}$. Thus $\left(\operatorname{rad} K^{*}\right) \cap R_{k}^{*}=\operatorname{rad}\left(K^{*} \cap R_{k}^{*}\right)=\operatorname{rad} R_{k}^{*}$, and so $\operatorname{rad} K^{*}=\operatorname{rad} A^{*}$. Hence $P^{*}$ contains every minimal prime ideal in $A^{*}$.

Conversely, let $K^{*}$ be as above, and define $I^{*}=K^{*} \cap R^{*}$. Then $R^{*} / I^{*}$ is a subring of $A_{P^{*}}^{*}$. Since $P^{*}$ contains all minimal prime ideals in $A^{*}$, $\operatorname{rad} I^{*}=\operatorname{rad}\left(K^{*} \cap R^{*}\right)=\left(\operatorname{rad} K^{*}\right) \cap R^{*}=\left(\operatorname{rad} A^{*}\right) \cap R^{*}$. Since $R^{*}$ is a subring of $A^{*},\left(\operatorname{rad} A^{*}\right) \cap R^{*}=\operatorname{rad} R^{*}$.

Also, since $K^{*}$ is an isolated ideal component of zero in $A^{*}$ and since $R_{k}^{*}$ and $A^{*}$ have the same total quotient ring, it follows that $I^{*}$ is an isolated ideal component of zero in $R^{*}$. And $A_{P^{*}}^{*}$ dominates $R^{*} / I^{*}$, since $P^{*} \cap R^{*}=M^{*}$. Hence, by Lemma $2, R$ is a quasi-subspace of $A_{P}$. Q.E.D.

REMARK 4. We give a number of known properties of unmixed and quasi-unmixed semilocal rings that will be needed in the remainder of the paper:

(1) $R$ is a quasi-unmixed semilocal ring if and only if $R / q$ is quasiunmixed and depth $q=$ altitude $R$, for every minimal prime divisor $q$ of zero in $R$ [4, Lemma 2.2].

(2) If $R$ is a quasi-unmixed semilocal ring and $P$ is a prime ideal in $R$, then $R_{P}$ is quasi-unmixed [4, Lemma 2.5].

(3) Let $R$ be a semilocal domain. If $R$ is quasi-unmixed and $A$ is a finitely generated domain over $R$, then $A$ is locally quasi-unmixed [4, Corollary 2.5].

(4) Let $(R, M)$ be a local ring. If altitude $R=0$, or altitude $R=1$ and $M$ is not a prime divisor of zero, then $R$ is unmixed and, therefore, quasi-unmixed.

3. Some characterizations of quasi-unmixed local rings. With Lemma 3 and Remark 4, the techniques of [6] can be adapted to prove most of the following results. The proofs are essentially accomplished by replacing "subspace" by "quasi-subspace", "unmixed" by "quasi-unmixed", "prime divisor of zero" by "minimal prime divisor of zero" and "Remark 4.6" by "Remark 4", and by making the appropriate reference changes. Since the proofs of Corollary 7 and Corollary 8 are entirely analogous to those in [6], they will be omitted.

Lemma 5 (CF. [6, Lemma 4.5(2)]). Let $R, R^{*}, A$ and $A^{*}$ be as in Lemma 2. Let $P$ be a prime ideal of $A$ such that $R$ is a quasi-subspace of $A_{1}$. Then the following statements hold:

(1) $P^{*}=P A^{*}$ is a prime ideal of $A^{*}$ that lies over $P$, and $A_{P}$ is a dense subspace of $A_{P *}^{*}$.

(2) $R$ is quasi-unmixed if and only if $A_{P}$ is quasi-unmixed. 
(3) If $Q$ is a prime ideal of $A$ such that $P \subseteq Q$, then $R$ is a quasi-subspace of $A_{Q}$.

Proof. By the domination of Definition 1, it is straightforward to show that $P \cap R=M$. (1) then follows by [6, Lemma 3.2]. It will be shown in Theorem 6(2)(a) that if $R$ is quasi-unmixed, then $A_{I}$, is quasi-unmixed (even if $R$ is not a quasi-subspace of $A_{P}$ ). The converse of (2) can be shown by using the quasi-unmixedness of $A_{P^{*}}^{*}$, (1) and Lemma 3 in an adaptation of the proo? in [6]. (3) is easily proved by using Lemma 3. Q.E.D.

The following theorem is the main result of this paper. It will be applied (Corollary 8) to characterize a quasi-unmixed ring $R$ in terms of quotient rings of certain Rees rings of $R$. Another application to a specific class of rings is given in Corollary 7 .

TheOREM 6 (CF. [6, THeOREM 4.1]). Let $(R, M)$ be a local ring with altitude $n \geqq 0$. Then:

(1) $R$ is quasi-unmixed if and only if there exist an integer $k$, elements $y_{1}, \cdots, y_{d}$ of the total quotient ring of $R_{k}$, and a prime ideal $P$ in $A=$ $R_{k}\left[y_{1}, \cdots, y_{d}\right]$ such that $R$ is a quasi-subspace of $A_{P}$ and $A_{P}$ is quasiunmixed.

(2) Let $f_{0}, f_{1}, \cdots, f_{d}$ be in $R_{k}(d \geqq 0$ and $k \geqq 0)$, where $f_{0}$ is not a zero divisor in $R_{k}$. Let $y_{i}=f_{i} \mid f_{0}$ and $A=R_{k}\left[y_{1}, \cdots, y_{d}\right]$. Then the following hold:

(a) If $R$ is quasi-unmixed, then $A$ is locally quasi-unmixed.

(b) If $P$ is a prime ideal in $R_{k}$ such that $\left(M, f_{0}, \cdots, f_{d}\right) R_{k} \subseteq P$ and such that $f_{0}, \cdots, f_{d}$ are a subset of a system of parameters in $R_{k P}$, then $P A$ is a prime ideal of $A$, height $P A=$ height $P-d$, and depth $P A=\operatorname{depth} P+d$.

(c) If $R$ is quasi-unmixed and $P$ is given in (b) then $R$ is a quasisubspace of $A_{Q}$, for all prime ideals $Q$ in $A$ such that $P A \subseteq Q$.

Proof. For (1), if $R$ is quasi-unmixed, then the conclusion will follow from (2). The converse follows by Lemma 5. (2)(b) is proven in [6] and is stated here for convenience. By using Remark 4, (2)(a) can be proven by adapting the proof in [6]. By noting that the proof of the fact that $R_{k P}$ is a dense subspace of $R_{k P *}^{*}$ in [6] is valid if $R$ is quasi-unmixed, (c) then follows by adapting the remainder of the proof in [6] and using the lemmas in this paper. Q.E.D.

Corollary 7 (CF. [6, Corollary 4.8]). Let $(R, M)$ be a local ring of altitude $n \geqq 1$. Assume that $M$ is not a prime divisor of zero. Then the following are equivalent:

(1) $R$ is quasi-unmixed.

(2) There exist analytically independent elements $x_{0}, x_{1}, \cdots, x_{n-1}$ in $R$ such that $x_{0}$ is not a zero-divisor and such that $R$ is a quasi-subspace of $A_{M_{A}}$, where $A=R\left[x_{1} / x_{0}, \cdots, x_{n-1} / x_{0}\right]$. 
(3) For every system of parameters $x_{0}, \cdots, x_{n-1}$ in $R$ such that $x_{0}$ is not a zero-divisor, $R$ is a quasi-subspace of $A_{M A}$, where $A$ is given in (2).

(4) There exists a finitely generated ring $A$ over $R$ such that $R \subseteq A \subseteq T$ where $T$ is the total quotient ring of $R$, and there exists a prime ideal $P$ in $A$ such that $R$ is a quasi-subspace of $A_{1}$, and $A_{1}$, is quasi-unmixed.

Let $B=\left(b_{1}, \cdots, b_{k}\right) R$ be an ideal in a Noetherian ring $R$. Let $t$ be an indeterminant, and let $u=1 / t$. The Rees ring $\mathscr{R}=\mathscr{R}(R, B)$ of $R$ with respect to $B$ is the ring $\mathscr{R}=R\left[u, t b_{1}, \cdots, t b_{k}\right]$. $\mathscr{R}$ is a graded Noetherian subring of $R[u, t]$. If $(R, M)$ is a local ring, then $\mathscr{M}=\left(M, u, t b_{1}, \cdots, t b_{k}\right)$ is the unique maximal homogeneous ideal of $\mathscr{R}$ [7, Theorem 3.1, step (ii)]. By [6, Remark 3.10(ii)], if $b_{1}, \cdots, b_{k}$ form a system of parameters in the local ring $(R, M)$, then $p=(M, u) \mathscr{R}$ is a height one depth $k$ prime ideal in $\mathscr{R}$, and $p$ is the radical of $u \mathscr{R}$ (and so $p$ is the unique height one prime divisor of $u \mathscr{R}$ ).

The characterization of certain concepts of a ring $R$ via the transition to a Rees ring has often been useful, and indeed this is the case here. Corollary 9 and the equivalence of (1) and (4) in Corollary 8 are the main results of this paper used in [8].

Corollary 8 (CF. [6, Corollary 4.9]). Let $(R, M)$ be a local ring of altitude $n \geqq 0$. The following are equivalent:

(1) $R$ is quasi-unmixed.

(2) There exist an ideal $B$ in $R$ and a prime ideal $P$ of $\mathscr{R}=\mathscr{R}(R, B)$ such that $R$ is a quasi-subspace of $\mathscr{R}_{P}$ and $\mathscr{R}_{P}$ is quasi-unmixed.

(3) There exists an ideal $B$ in $R$ such that $\mathscr{R}_{\mathscr{M}}$ is quasi-unmixed, where $\mathscr{R}=\mathscr{R}(R, B)$ and $\mathscr{M}$ is the maximal homogeneous ideal of $\mathscr{R}$.

(4) For every ideal $B$ of $R$ that is generated by a system of parameters, $R$ is a quasi-subspace of $\mathscr{R}_{(, u, u) \mathscr{R}}$, where $\mathscr{R}=\mathscr{R}(R, B)$.

$\left(4^{\prime}\right)$ There exists an ideal $B$ of $R$ that is generated by a system of parameters such that $R$ is a quasi-subspace of $\mathscr{R}_{(, u, u) \mathscr{R}}$, where $\mathscr{R}=\mathscr{R}(R, B)$.

COROllary 9. Let $(R, M)$ be a local ring with completion $\left(R^{*}, M^{*}\right)$. Let $B$ be an $M$-primary ideal of $R$ that is generated by a system of parameters. Let $\mathscr{R}=\mathscr{R}(R, B)$ and $\mathscr{R}^{*}=\mathscr{R}\left(R^{*}, B R^{*}\right)$. Then $R$ is quasi-unmixed if and only if $\left(M^{*}, u\right) \mathscr{R}^{*}$ contains all minimal prime divisors of zero in $\mathscr{R}^{*}$.

Proof. Use Corollary $8\left((1)\right.$ and $\left.\left(4^{\prime}\right)\right)$ and Lemma 3. Q.E.D.

\section{BIBLIOGRAPHY}

1. D. Ferrand and M. Raynaud, Fibres formelles d'un anneau local Noethérien, Ann. Sci. École Norm. Sup. (4) 3 (1970), 295-311. MR 42 \#7660.

2. M. Nagata, On the chain problem of prime ideals, Nagoya Math. J. 10 (1965), 51-64. MR 18, 8. 
3. M. Nagata, Local rings, Interscience Tracts in Pure and Appl. Math., no. 13, Interscience, New York, 1962. MR 27 \#5790.

4. L. J. Ratliff, Jr., On quasi-unmixed semi-local rings and the altitude formula, Amer. J. Math. 87 (1965), 278-284. MR 31 \#3448.

5. - On quasi-unmixed local domains, the altitude formula, and the chain condition for prime ideals. I, Amer. J. Math. 91 (1969), 508-528. MR 40 \#136.

6. - On quasi-unmixed local domains, the altitude formula, and the chain condition for prime ideals. II, Amer. J. Math. 92 (1970), 99-144. MR 42 \#249.

7. D. Rees, A-transforms of local rings and a theorem on multiplicities of ideals, Proc. Cambridge Philos. Soc. 57 (1961), 8-17. MR 22 \#9521.

8. P. Sawtelle, Characterizations of unmixed and quasi-unmixed local domains, Ph.D. Dissertation, University of California at Riverside, Riverside, California, 1971.

9. O. Zariski and P. Samuel, Commutative algebra. Vol. I, University Series in Higher Math., Van Nostrand, Princeton, N.J., 1958. MR 19, 833.

Department of Mathematics, University of Missouri-Rolla, Rolla, Missouri 65401 